\title{
Procedimento para avaliação da resiliência de sistemas de transportes
}

\author{
A procedure for the evaluation of the resilience of transportation systems
}

\section{Camila Maestrelli Leobons ${ }^{1}$, Vânia Barcellos Gouvêa Campos², Renata Albergaria de Mello Bandeira ${ }^{3}$}

1Instituto Militar de Engenharia, camila@maestrelli.org

2Instituto Militar de Engenharia, vania@ime.eb.br

3Instituto Militar de Engenharia, re.albergaria@gmail.com

\section{Recebido:}

2 de maio de 2019

Aceito para publicação:

9 de janeiro de 2020

Publicado:

31 de agosto de 2020

Editor de área:

Bruno Vieira Bertoncini

\section{Palavras-chaves:}

Resiliência.

Sistemas de Transportes.

Indicadores.

Keywords:

Resilience.

Transportation systems.

Indicators

DOI:10.14295/transportes.v28i3.2038

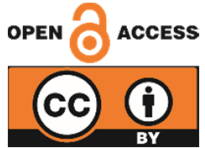

\begin{abstract}
RESUMO
Neste trabalho, propõe-se uma medida de resiliência de sistemas de transportes, que está relacionada à capacidade destes em reduzir o impacto na movimentação de pessoas em uma cidade após um evento, climático ou causado pelo homem, que gere perturbações em partes destes sistemas. Uma interrupção dos serviços, em um determinado modo de transportes, sobrecarrega outros modos que, não tendo capacidade de absorver, impactam a mobilidade na cidade, gerando grandes inconvenientes para a população. Nesse contexto, indicadores e índices representam um importante instrumento de monitoramento do nível de resiliência. Assim, o objetivo deste artigo consiste no desenvolvimento de um procedimento para caracterização do nível de resiliência da rede de transportes de uma cidade, que contempla os diferentes modos de transporte oferecidos na cidade e compara as resiliências das zonas da cidade. Para confirmar a aplicabilidade do procedimento desenvolvido, a cidade do Rio de Janeiro é selecionada para estudo de caso.
\end{abstract}

\section{ABSTRACT}

This paper proposes a resilience measure of transportation systems, which is related to their ability to reduce the impact on the movement of passengers and freight in a city after a climatic or man-made event that causes disturbances in these systems. A service interruption in a certain mode can lead to the overload other modes that, not being able to absorb, can impact the level of mobility in a city, causing considerable inconvenience to the population. In this context, indicators and indexes are important tools to monitor the level of resilience. Therefore, this study proposes a procedure to characterize the level of resilience of an urban transportation network, which integrates the different modes of transportation offered in the city and compares the resilience of the city's zones. Finally, to confirm the applicability of the procedure created, the city of Rio de Janeiro is selected for the development of a case study.

\section{INTRODUÇÃO}

Um sistema de transportes é de fundamental importância para a atividade diária de uma cidade, de modo que qualquer redução em seu desempenho pode comprometer a operação de diversos setores, prejudicando serviços de emergência, entregas, acesso ao trabalho, entre outros, afetando o mercado interno e a economia, bem como a qualidade de vida da população (A. S. Santos, 2014). Entretanto, todo sistema de transporte urbano pode estar sujeito a choques repentinos, rotas que cruzam a cidade podem ser bloqueadas ou danificadas por tempestades, inundações, ou mesmo por eventos recorrentes, como congestionamento e acidentes. Por isso, as cidades devem estar constantemente melhorando seu sistema de transportes para suportar tais ocorrências, buscando assim a resiliência. 
A preocupação com a resiliência em cidades veio da necessidade de "reagir" aos impactos do crescimento populacional e de lidar com incertezas e desafios de ameaças ao funcionamento normal da cidade. 0 termo resiliência em cidades é recente, tendo sido introduzido por Godschalk (2003), cujo trabalho focou em perigos urbanos. Entretanto, a resiliência já havia sido definida no contexto de desastres em comunidades por Timmerman (1981), Comfort (1999) e Mileti (1999) como a capacidade de lidar com os perigos à dinâmica da cidade, especialmente àqueles relacionados à mudança climática (Cartalis, 2014). Ainda hoje, mesmo com diversas ações para sua implementação, não existe um consenso em relação à definição do conceito de resiliência (Meerow et al., 2016).

Enquanto Timmerman (1981) define resiliência como a capacidade de sistemas em absorver e recuperar, Comfort (1999) aponta para a adaptação a novas condições e, Mileti (1999) destaca a resistência sem auxílio externo à comunidade. Bruneau et al. (2003) agregaram as três definições ao caracterizar resiliência como a capacidade de unidades sociais em mitigar perigos, conter seus efeitos negativos a fim de minimizar perdas sociais e econômicas, e executar atividades de recuperação para restaurar o nível "normal" de desempenho do sistema (não necessariamente igual ao anterior). Para Bruneau et al. (2003), a resiliência de um sistema pode ser medida por meio de um conjunto multidimensional de indicadores de desempenho em função de propriedades determinantes de um sistema resiliente. Tais propriedades foram apresentadas pela estrutura 4R: Robustez, Redundância, Recursos e Rapidez.

Em transportes, a Robustez reflete a capacidade da rede e seus elementos em suportar o impacto de eventos de ruptura sem degradação significativa no desempenho do sistema. Redundância é a medida em que as rotas alternativas e modos de transporte podem ser empregados se alguns componentes estão danificados. A dimensão Recursos está relacionada à disponibilidade de materiais, suprimentos e equipes para restaurar a funcionalidade. Por sua vez, Rapidez é a capacidade para restaurar a funcionalidade em tempo hábil, sendo assim, resultado dos outros três (Tierney e Bruneau, 2007).

A resiliência para sistemas de transportes pode ser entendida, sob o ponto de vista da mobilidade, como a capacidade em mantê-la em níveis satisfatórios, bem como o restabelecimento de sua normalidade mesmo diante de colapsos. Isto posto, a mobilidade pode variar em consequência de uma perturbação no sistema, o que pode provocar a impossibilidade de se restabelecer a situação pós-evento de forma rápida, mesmo que seja com um nível mais baixo de serviço.

Assim, como identificar a possibilidade de uma "imobilidade" ocorrer em consequência de um de evento? Uma região com um bom nível de mobilidade pode ter um baixo nível de resiliência nos transportes, o que, diante de uma perturbação, favorece uma situação de colapso. Por outro lado, uma região pode ter uma baixa mobilidade, porém, um bom nível de resiliência nos transportes. Isto se deve à estrutura do próprio sistema de transporte, que pode apresentar características que facilitem uma mudança nas rotas de deslocamento, capacidade estocada e um bom plano de adaptação a situações adversas.

Visando um sistema de transportes mais resiliente como uma forma de reduzir os impactos de uma ameaça, indicadores e índices constituem uma ferramenta importante para avaliar o nível de resiliência, de modo que o objetivo deste trabalho é propor um procedimento para medir a resiliência de sistemas de transportes em condições pré-evento, relacionada com a infraestrutura e a operação do sistema, independente do risco e tipo de evento, por meio de um ín- 
dice de resiliência. Assim, na seção 2 é apresentada uma revisão da literatura, abordando o conceito de resiliência em sistemas de transportes, bem como uma discussão sobre processos de avaliação da resiliência desses sistemas. A seção 3 propõe um conjunto de atributos e indicadores que devem ser considerados para a avaliação da resiliência de sistemas de transportes. Na seção 4, apresenta-se a proposta de procedimento para caracterização do nível de resiliência da rede de transportes numa cidade. Na seção 5, esse procedimento é aplicado na cidade do Rio de Janeiro, sendo os resultados analisados na seção 6. Enfim, na seção 7 apresentam-se as considerações finais.

\section{REVISÃO DA LITERATURA}

Com o intuito de documentar o estado da arte em relação à avaliação da resiliência em transportes, uma revisão sistemática da literatura (RSL) foi realizada, seguindo a metodologia proposta por Thomé et al. (2016). A busca, realizada na Scopus e Web of Science, adotou os seguintes grupos de palavras-chave: (1) transport* AND resilien*; (2) urban OR city; (3) variable OR indicator OR metric OR index. 0 primeiro grupo destaca a resiliência em transportes, o segundo restringe ao transporte urbano, enquanto o terceiro limita a busca para indicadores na área em estudo.

Essa pesquisa retornou 105 artigos. Porém, após a leitura dos títulos e resumo, verificou-se que apenas 26 artigos focavam em resiliência em transportes. Após leitura do texto completo, foram excluídos mais 20 artigos, por não estarem relacionados à avaliação da resiliência. Enfim, 29 publicações foram incluídas pelo processo snowball, totalizando 35 trabalhos analisados. Nesse processo, alguns trabalhos de resiliência em escala regional também foram adicionados por serem relevantes na literatura, devido ao grande número de citações.

Em relação ao conceito de resiliência em transportes, as definições apresentadas revelaram uma inconsistência na adoção de um conceito único, porém, verifica-se que tais definições focam em uma ou mais capacidades de um sistema resiliente: capacidade de resistir (Adams et al., 2012; Faturechi e Miller-Hooks, 2014); na capacidade de absorver o impacto da perturbação (Adjetey-Bahun et al., 2016; Arcidiacono et al., 2012; Faturechi e Miller-Hooks, 2014; Freckleton et al., 2012; Ta et al., 2009; Tierney e Bruneau, 2007; VTPI, 2017); na capacidade de manter o fornecimento do serviço (Ortiz et al., 2009; Ta et al., 2009); e na rápida recuperação das funções normais (Adams et al., 2012; Adjetey-Bahun et al., 2016; Arcidiacono et al., 2012; Balal et al., 2017; Chan e Schofer, 2016; Chen e Miller-Hooks, 2012; Faturechi e Miller-Hooks, 2014; Freckleton et al., 2012; Murray-Tuite, 2006; Ortiz et al., 2009; Tierney e Bruneau, 2007). Logo, considerando que as capacidades de resistir e absorver o impacto estão relacionadas à capacidade de preservação da oferta do serviço, nota-se que a resiliência em transportes está relacionada à continuidade do serviço prestado ou à recuperação das atividades após um evento, retornando à normalidade ou próximo desta numa região.

Os trabalhos revisados também podem ser agrupados em relação à visão temporal, segundo Rose $(2004,2007)$, em estáticos ou dinâmicos. As avaliações estáticas se restringem a um determinado ponto no tempo, enquanto as dinâmicas levam em consideração a variação no tempo. Os trabalhos de quantificação da resiliência com dados reais (Adams et al., 2012; Chan e Schofer, 2016; Cox et al., 2011; Donovan e Work, 2017) estão na categoria dinâmica. Além destes, Vugrin et al. (2010), Arcidiacono et al. (2012), Pant (2012), Adjetey-Bahun et al. (2016) e Balal et al. (2017) examinaram a resiliência dinamicamente, enquanto D’Lima e Medda (2015) 
apresentaram um método para estimação da rapidez de recuperação do sistema. Beiler et al. (2013) também considerou a variação temporal de indicadores do sistema, porém, analisandoos historicamente.

Ainda, a revisão detectou um maior número de estudos contemplando um único modo de transporte, sendo o rodoviário o mais discutido, e grande parte destes adotaram modelos de alocação do tráfego para avaliação da resiliência. Em relação aos indicadores, os mais utilizados foram: tempo ou distância de viagem, relação entre a demanda e capacidade, disponibilidade de recursos financeiros para ações de resiliência e de melhoria da resiliência.

Quanto à escala de análise, foi observado que a avaliação de redes foi a mais explorada na literatura até o momento, prevalecendo modelos de otimização. Outra técnica muito empregada para avaliação de cenários compreende a remoção de links ou nós, que, em alguns casos (King e Shalaby, 2016; Leu et al., 2010; Omer et al., 2013; Pant, 2012), resultou na identificação de componentes críticos do sistema.

As análises que apresentaram modelos de otimização forneceram, em sua maioria, uma unificação da medida da resiliência, cujas quantificações predominantes foram em função do tempo de viagem e da demanda (não) atendida, considerando a resiliência como a fração de um indicador antes e após um evento perturbador. As abordagens de Serulle et al. (2011) e Freckleton et al. (2012), por meio da Lógica Fuzzy, e a de Hughes e Healy (2014), por estrutura semiquantitativa, também geraram uma unificação da resiliência, contudo, por meio de uma agregação de indicadores. Porém, dentre todas as publicações (qualitativas e quantitativas) apenas quatro chegaram a um nível de resiliência: Adams et al. (2012), Serulle et al. (2011), Freckleton et al. (2012), e Hughes e Healy (2014). 0 primeiro analisou a resiliência em um segmento de um corredor rodoviário após eventos climáticos, não chegando numa unificação de atributos para definir um valor de resiliência para o corredor. Os três últimos propuseram um índice de resiliência com o uso de diversos indicadores sem avaliar a rede objetivamente. Enquanto Serulle et al. (2011) e Freckleton et al. (2012) adotaram a lógica fuzzy para a mensuração da resiliência com o uso de bons indicadores, porém, medidos de forma superficial, Hughes e Healy (2014) propuseram indicadores mais genéricos, sem uma quantificação, estes que foram apenas graduados numa escala de 1 a 4, numa comparação de cenários de eventos, onde 1 indica o pior caso e 4 o melhor cenário.

No Brasil, os trabalhos de resiliência na área de transportes focam em sua maioria na restrição de combustíveis. Fernandes et al. (2017) apresenta o termo "resiliência da mobilidade" sob o ponto de vista da limitação da disponibilidade de recursos energéticos, como petróleo e gás. Para Fernandes et al. (2017), a análise da resiliência da mobilidade urbana deve avaliar três etapas: a capacidade de resistir, de se adaptar diante de um risco à mobilidade e o potencial de se transformar para a melhoria da mobilidade urbana.

Neste mesmo contexto de redução da oferta de combustíveis, Martins e Silva (2018) apresentaram uma proposta para avaliação da resiliência da mobilidade urbana considerando cenários de restrição ao transporte motorizado. Foi utilizado como parâmetro as distâncias máximas possíveis (DMP) entre as zonas de tráfego. Segundo os autores, essa distância é inversamente proporcional à possibilidade do deslocamento em transportes ativos, como a pé ou bicicleta. Dessa forma, quanto menor a distâncias entre as zonas de origem e destino, maior a resiliência da mobilidade entre as regiões. Azolin e Silva (2019) ampliaram o estudo de Martins e Silva (2018) ao acrescentar a possibilidade do deslocamento por transporte público, tendo este uma oferta limitada. 
Sob outra perspectiva, Santos (2014) apresenta um plano de resiliência em transportes frente às mudanças climáticas, considerando como elementos-chave a governança, a informação, os recursos financeiros e o uso de indicadores como ferramenta para verificação da eficácia das ações.

Ázaro (2017) propôs um índice para avaliação da resiliência de linhas de ônibus urbanos das cidades brasileiras por meio de análise multicritério considerando como indicadores o tamanho da frota, a extensão da linha, a frequência/ intervalo entre os ônibus da linha e o percentual da extensão da linha em faixas/corredores exclusivos.

Santos et al. (2018) utilizaram a lógica fuzzy para analisar a resiliência entre regiões frente à ausência de integração tarifária, considerando como variáveis a população da origem, quantidade de empregos, oportunidades intervenientes entre as regiões, tempo de viagem, diferença tarifária (economia com a integração para o usuário) e renda da origem, observando que as origens com maior renda e transporte de alta capacidade possuem maior resiliência.

Enfim, com base na revisão bibliográfica, buscou-se identificar os principais indicadores de resiliência discutidos na literatura acadêmica de transportes e como esses poderiam ser tratados de forma a servir de instrumento de planejamento de transportes de uma região visando a resiliência dos sistemas de transportes.

\section{PROPOSTA DE ATRIBUTOS E INDICADORES}

Para a proposta de atributos e indicadores, partiu-se da premissa de que poderiam ser associados, gerando uma medida de resiliência a ser utilizada para evidenciar riscos de interrupção da movimentação de pessoas e de carga numa região. Tais riscos podem estar relacionados à própria infraestrutura ou à operação dos sistemas. Quanto à infraestrutura, a resiliência está relacionada à conectividade da rede, número de rotas alternativas, e pontos estratégicos/críticos.

Outra visão está relacionada à operação dos sistemas, em que a capacidade de determinado modo de transporte pode ser afetada, limitando o nível de serviço. Neste caso, é importante considerar o deslocamento de pessoas em transportes públicos, uma vez que uma interrupção dos serviços em um determinado modo, especialmente em um transporte de massa, sobrecarrega outros sistemas que, não tendo capacidade de absorver, podem impactar a mobilidade numa cidade. Ressalta-se que um problema na infraestrutura do sistema provocaria um impacto em sua operação.

Ainda, com base na análise dos artigos levantados na RSL identificaram-se dois direcionamentos de análise: um relacionado à rede estrutural, rodo e metroviária, e outro vinculado à operação dos serviços oferecidos, considerando a capacidade e a demanda de transporte. Logo, embasado na ideia de que os atributos e indicadores utilizados em estudos anteriores podem ser empregados de forma mais objetiva e consistente, e considerando as duas visões de análise (infraestrutura e operação dos sistemas), um conjunto de 11 atributos e indicadores é proposto, fundamentado nas propriedades R4 (Robustez, Redundância, Recursos e Rapidez) apontadas por Bruneau et al. (2003). Na Tabela 1, é apresentada uma proposta de atributos e indicadores que podem ser utilizados para medir resiliência de um sistema de transportes com base no R4. Essa proposta sintetiza o que foi observado na literatura na análise da resiliência. 
Tabela 1 - Proposta de atributos e indicadores de resiliência em transportes

\begin{tabular}{|c|c|c|c|c|}
\hline Propriedade & Infraestrutura & Operação & Atributos e Indicadores & Autores \\
\hline \multirow{5}{*}{ Robustez } & \multirow{5}{*}{$\begin{array}{l}\text { Topologia da rede } \\
\text { (vias ou interseções) }\end{array}$} & \multirow{5}{*}{$\begin{array}{l}\text { Capacidade/ } \\
\text { demanda }\end{array}$} & $\begin{array}{l}\text { Conectividade/ Vulnerabili- } \\
\text { dade da Rede }\end{array}$ & $\begin{array}{l}\text { Beiler et al., 2013; Freckleton et al., 2012; Jin et al., } \\
\text { 2014; King e Shalaby, 2016; Leu et al., 2010; Serulle et } \\
\text { al., 2011; Zhang et al., } 2015\end{array}$ \\
\hline & & & Capacidade Viária & $\begin{array}{l}\text { Adams et al., 2012; Balal et al., 2017; Bekkem et al., } \\
\text { 2011; Bhavathrathan e Patil, 2015; Chen e Miller- } \\
\text { Hooks, 2012; Faturechi e Miller-Hooks, 2014b, 2014c; } \\
\text { Fotouhi et al., 2017; Freckleton et al., 2012; King e } \\
\text { Shalaby, 2016; Miller-Hooks et al., 2012; Murray- } \\
\text { Tuite, 2006; Omer et al., 2013; Pant, 2012; Patil e } \\
\text { Bhavathrathan, 2016; Serulle et al., 2011; Vugrin et } \\
\text { al., 2010; Ye e Ukkusuri, 2015; Zhang e Miller-Hooks, } \\
\text { 2015; Zhang et al., } 2015\end{array}$ \\
\hline & & & $\begin{array}{l}\text { Capacidade do Transporte } \\
\text { Coletivo de Massa }\end{array}$ & $\begin{array}{l}\text { Adjetey-Bahun et al., 2016; Cox et al., 2011; D'Lima e } \\
\text { Medda, 2015; Jin et al., 2014; Pant, } 2012\end{array}$ \\
\hline & & & Demanda & $\begin{array}{l}\text { Adams et al., 2012; Adjetey-Bahun et al., 2016; } \\
\text { Bekkem et al., 2011; Bhavathrathan e Patil, 2015; } \\
\text { Chan e Schofer, 2016; Chen e Miller-Hooks, 2012; Cox } \\
\text { et al., 2011; D'Lima e Medda, 2015; Faturechi e Miller- } \\
\text { Hooks, 2014b, 2014c; Fotouhi et al., 2017; Freckleton } \\
\text { et al., 2012; Jin et al., 2014; King e Shalaby, 2016; Liu e } \\
\text { Murray-Tuite, 2008; Miller-Hooks et al., 2012; } \\
\text { Murray-Tuite, 2006; Omer et al., 2013; Pant, 2012; } \\
\text { Patil e Bhavathrathan, 2016; Serulle et al., 2011; } \\
\text { Vugrin et al., 2010; Ye e Ukkusuri, 2015; Zhang e } \\
\text { Miller-Hooks, 2015; Zhang et al., } 2015\end{array}$ \\
\hline & & & Tempo de Viagem & $\begin{array}{l}\text { Adjetey-Bahun et al., 2016; Balal et al., 2017; Beiler et } \\
\text { al., 2013; Bhavathrathan e Patil, 2015; Donovan e } \\
\text { Work, 2017; Faturechi e Miller-Hooks, 2014c; Fotouhi } \\
\text { et al., 2017; Freckleton et al., 2012; Jin et al., 2014; } \\
\text { King e Shalaby, 2016; Liu e Murray-Tuite, 2008; } \\
\text { Murray-Tuite, 2006; Omer et al., 2013; Pant, 2012; } \\
\text { Patil e Bhavathrathan, 2016; Serulle et al., 2011; } \\
\text { Vugrin et al., } 2010\end{array}$ \\
\hline \multirow{3}{*}{ Redundância } & \multirow{3}{*}{ Rotas alternativas } & \multirow{3}{*}{$\begin{array}{l}\text { Modos de } \\
\text { transportes }\end{array}$} & $\begin{array}{l}\text { Rotas Alternativas em Rela- } \\
\text { ção aos Pontos Críticos }\end{array}$ & $\begin{array}{l}\text { Balal et al., 2017; Bekkem et al., 2011; Cox et al., } \\
\text { 2011; Freckleton et al., 2012; Jin et al., 2014; King e } \\
\text { Shalaby, 2016; Leu et al., 2010; Murray-Tuite, 2006; } \\
\text { Pant, 2012; Serulle et al., 2011; Ye e Ukkusuri, } 2015\end{array}$ \\
\hline & & & Nível de Acessibilidade & $\begin{array}{l}\text { Arcidiacono et al., 2012; Beiler et al., 2013; Donovan e } \\
\text { Work, 2017; Freckleton et al., 2012; King e Shalaby, } \\
\text { 2016; Leu et al., 2010; Liu e Murray-Tuite, 2008; } \\
\text { Murray-Tuite, 2006; Omer et al., 2013; Pant, 2012; } \\
\text { Serulle et al., 2011; Zhang et al., } 2015\end{array}$ \\
\hline & & & Modos Concorrentes & $\begin{array}{l}\text { Adjetey-Bahun et al., 2016; Chen e Miller-Hooks, } \\
\text { 2012; Freckleton et al., 2012; Jin et al., 2014; King e } \\
\text { Shalaby, 2016; Leu et al., 2010; Miller-Hooks et al., } \\
\text { 2012; Omer et al., 2013; Pant, 2012; Serulle et al., } \\
\text { 2011; Ye e Ukkusuri, } 2015\end{array}$ \\
\hline \multirow[b]{2}{*}{ Recursos } & \multirow[b]{2}{*}{$\begin{array}{l}\text { Equipamentos e pes- } \\
\text { soas }\end{array}$} & \multirow[b]{2}{*}{$\begin{array}{l}\text { Reserva de ati- } \\
\text { vos e pessoas }\end{array}$} & $\begin{array}{l}\text { Tempo para Início do Pro- } \\
\text { cesso de Recuperação do Im- } \\
\text { pacto }\end{array}$ & $\begin{array}{l}\text { Chan e Schofer, 2016; Freckleton et al., 2012; Liu e } \\
\text { Murray-Tuite, 2008; Murray-Tuite, 2006; Zhang e } \\
\text { Miller-Hooks, } 2015\end{array}$ \\
\hline & & & $\begin{array}{l}\text { Disponibilidade de Pessoal e } \\
\text { Recursos para Agir }\end{array}$ & $\begin{array}{l}\text { Adjetey-Bahun et al., 2016; Arcidiacono et al., 2012; } \\
\text { Chen e Miller-Hooks, 2012; Faturechi e Miller-Hooks, } \\
\text { 2014b, 2014c; Fotouhi et al., 2017; Freckleton et al., } \\
\text { 2012; Jin et al., 2014; Miller-Hooks et al., 2012; Omer } \\
\text { et al., 2013; Pant, 2012; Serulle et al., 2011; Vugrin et } \\
\text { al., 2010; Ye e Ukkusuri, 2015; Zhang e Miller-Hooks, } \\
2015\end{array}$ \\
\hline Rapidez & Dimensão do Evento & $\begin{array}{l}\text { Qualidade da } \\
\text { equipe, recur- } \\
\text { sos e informa- } \\
\text { ção }\end{array}$ & $\begin{array}{l}\text { Tempo para Atingir a Norma- } \\
\text { lidade da Operação ou Pró- } \\
\text { ximo desta }\end{array}$ & $\begin{array}{l}\text { Adams et al., 2012; Adjetey-Bahun et al., 2016; Arcidi- } \\
\text { acono et al., 2012; Balal et al., 2017; Chan e Schofer, } \\
\text { 2016; Chen e Miller-Hooks, 2012; D'Lima e Medda, } \\
\text { 2015; Donovan e Work, 2017; Faturechi e Miller- } \\
\text { Hooks, 2014b, 2014c; Fotouhi et al., 2017; Jin et al., } \\
\text { 2014; Miller-Hooks et al., 2012; Murray-Tuite, 2006; } \\
\text { Omer et al., 2013; Pant, 2012; Vugrin et al., 2010; Ye e } \\
\text { Ukkusuri, 2015; Zhang e Miller-Hooks, 2015; Zhang et } \\
\text { al. } 2015\end{array}$ \\
\hline
\end{tabular}




\section{PROCEDIMENTO PARA DEFINIÇÃO DO NÍVEL DE RESILIÉNCIA DE UMA REGIÃO}

Na proposta do índice de resiliência, considerou-se que Robustez e Redundância estão diretamente relacionadas ao sistema de transportes e à sua capacidade em manter um nível de serviço aceitável, enquanto Rapidez e Recursos estão associados à gravidade do evento, à disponibilidade financeira e de pessoas envolvidas, tanto para operadores quanto na municipalidade.

A resiliência intrínseca do sistema de transportes pode ser entendida como a capacidade em manter o nível de mobilidade em padrões aceitáveis. Portanto, considera-se que a resiliência própria do sistema pode ser calculada em função da Robustez e da Redundância, identificando os aspectos físicos e operacionais que devem ser melhorados a fim de reduzir o impacto causado na mobilidade por um evento qualquer. Dessa forma, com a finalidade de contribuir para o planejamento da mobilidade, especialmente quanto às possibilidades de emergências, propõe-se um procedimento para análise da resiliência em sistemas de transportes por meio do desenvolvimento de um Índice de Resiliência em função dos indicadores de Robustez e Redundância.

A partir da revisão da literatura apresentada na seção 2, constatou-se a inexistência de estudos analisando a resiliência urbana entre regiões, integrando os diferentes modos de transporte existentes em uma cidade e considerando a influência da interrupção de um determinado modo de transporte sobre os demais. Assim, o procedimento proposto visa preencher essa lacuna da literatura, permitindo a avaliação da resiliência entre regiões da cidade, bem como da cidade como um todo, contemplando a interdependência dos diferentes modos de transporte urbano, possibilitando a comparação dos índices para a identificação de áreas com menor resiliência e que demandam prioridade de investimento em transporte. Logo, este procedimento dá suporte ao planejamento de transporte, visando uma maior mobilidade na região estudada, de modo a evitar maiores transtornos ao deslocamento da população no caso de um desastre ou evento negativo para o sistema.

A estrutura metodológica proposta é composta por cinco etapas: (I) Reconhecimento da rede; (II) Medição dos Indicadores; (III) Cálculo do Índice de Resiliência; (IV) Cálculo da Resiliência de uma região e (V) Determinação do nível de resiliência, detalhadas a seguir.

\subsection{Etapa 1: Reconhecimento da Rede}

Esta etapa consiste na definição da região em análise, delimitação de suas sub-regiões e mapeamento dos principais corredores interligando essas regiões, tanto individual quanto coletivo, entre os pares origem-destino. A delimitação das sub-regiões pode ser determinada a partir de uma demarcação pré-existente, podendo ser definida por regiões administrativas ou áreas de planejamento. Essas sub-regiões representam as zonas de tráfego de acordo com as matrizes origens/destinos com dados atuais ou futuros. Ainda, são identificados os modos de transportes oferecidos pela cidade e suas respectivas redes são observadas. Dessa forma, é fundamental a obtenção da rede georreferenciada representativa da região em análise e as matrizes de viagens por modo individual e coletivo.

\subsection{Etapa 2: Medição dos Indicadores}

Conforme previamente mencionado, para medir a resiliência foram selecionados indicadores de Robustez e Redundância, pois estão diretamente relacionados à estrutura e operação dos 
sistemas, quais sejam: Modos de transportes, Rotas independentes, Vulnerabilidade da Rede, Demanda de viagem entre regiões, Capacidade e Demanda de passageiros por modo e Tempo de Viagem. Na Tabela 2, apresenta-se a forma de quantificá-los.

Tabela 2 - Quantificação dos indicadores para composição do índice de Resiliência

\begin{tabular}{l|l}
\hline INDICADORES & QUANTIFICAÇÃO \\
\hline Modos de transportes concorrentes & Sistemas de transportes disponíveis entre um par de O/D \\
\hline $\begin{array}{l}\text { Número de rotas independentes } \\
\text { por modo de transporte }\end{array}$ & $\begin{array}{l}\text { Numero de rotas independentes entre um par de origem e destino. Fundamenta-se no } \\
\text { Método para identificação do Subconjunto de Articulação Minimal (MAS - Minimal } \\
\text { Articulation Subset) que permite identificar o número de caminhos disjuntos, apresentado } \\
\text { em CAMPOS; SILVA (2004). }\end{array}$ \\
\hline Vulnerabilidade da Rede & $\begin{array}{l}\text { V=1/n, onde n é o numero de rotas independentes por modo entre um par de origem e } \\
\text { destino }\end{array}$ \\
\hline Capacidade Viária & $\begin{array}{l}\text { Capacidade horária por rota (veiculos por hora na via), com base em dados de centros de } \\
\text { controle de tráfego ou utilizando os métodos do HCM (Highway Capacity Manual) }\end{array}$ \\
\hline Capacidade do Transporte Coletivo \\
de Massa & $\begin{array}{l}\text { Oferta de lugares ( passageiros em pé e sentado), com base em número de linhas em } \\
\text { circulação por rota (rodo e metroferroviária) dada por operadores de sistemas de } \\
\text { transporte público }\end{array}$ \\
\hline Demanda & $\begin{array}{l}\text { Estabelecida por meio de Pesquisas Origem-Destino, podendo ser encontradas em Planos } \\
\text { de Transporte ou levantadas em campo (Viagens (pessoas) entre O/D). }\end{array}$ \\
\hline Tempo de Viagem & $\begin{array}{l}\text { O tempo de deslocamento por rota alternativa na hora de pico de um ponto inicial até o } \\
\text { destino final. Pode ser obtido a partir de pesquisas de campo ou de informações de } \\
\text { operadores dos sistemas públicos . }\end{array}$ \\
\hline
\end{tabular}

A Vulnerabilidade da Rede está vinculada à infraestrutura da rede, ao passo que a Capacidade e a Demanda de passageiros por modo, bem como o Tempo de Viagem, estão associados à operação do sistema. Ainda, a demanda é calculada tanto em relação à quantidade de viagens entre a zona de origem e a zona de destino, quanto à demanda de fluxo de veículos nas vias da rede e de passageiros no modo coletivo por hora de pico. Assim, a demanda referente à quantidade de viagens entre as regiões é determinada a partir da Matriz Origem-Destino do modo em questão, enquanto a medição dos indicadores restantes é quantificada por meio da análise da rede.

Entre cada par de origem e destino o tempo de viagem, a capacidade e a demanda de passageiros de um determinado modo são definidas, assim como a média do Tempo de Viagem, a soma das capacidades e do volume de passageiros das rotas alternativas, respectivamente. Logo, os indicadores são obtidos para cada uma das rotas alternativas para cada modo de transporte entre cada par de origem-destino.

\subsection{Etapa 3: Método de Cálculo do Índice}

Esta etapa compreende o cálculo da resiliência entre regiões, a resiliência relativa que possibilitará a medição das resiliências de cada região na etapa seguinte. Para isso, foi definido que a resiliência está relacionada ao que foi chamado de "custo de desempenho". Esse custo é calculado para cada modo de transporte e representa uma relação entre a demanda pelo modo em análise e as "folgas" em sistemas concorrentes entre um par origem-destino, e a vulnerabilidade desse modo, conforme apresentado na Equação 1.

$$
C D_{i j}^{M A}=\frac{D_{i j}^{M A}}{\sum_{m \neq M A} C E X_{i j}^{m}} x \frac{\frac{\sum_{m \neq M A} t_{i j}^{m}}{m_{i j}-1}}{t_{i j}^{M A}} x V_{i j}^{M A}
$$

onde: $\quad$ i: $\quad$ Zona de Origem;

j: $\quad$ Zona de Destino.

MA: $\quad$ Modo de transporte em análise entre um par de O/D (i, j); 
m: $\quad$ Modo de transporte, onde: $\mathrm{m}$ = rodoviário individual, rodoviário coletivo, metroferroviário ou outro;

$\mathrm{m}_{\mathrm{ij}}$ : $\quad$ Número de modos de transporte entre as regiões i e j;

$C D_{i j}^{M A}$ : $\quad$ Custo de Desempenho do modo de transporte em análise (MA) entre a origem i e o destino j;;

$C E X_{i j}^{m}$ : $\quad$ Capacidade residual (folga) do modo de transporte $\mathrm{m}$ entre a origem $\mathrm{i}$ e o destino j (diferença entre a capacidade total e a demanda existente)

$t_{i j}^{m}$ : Tempo médio de viagem de cada modo (concorrente) de transporte m entre a origem i e o destino j;

$t_{i j}^{M A}$ : Tempo médio de viagem do modo de transporte em análise (MA) entre a origem i e o destino j;

$D_{i j}^{M A}: \quad$ Demanda de viagens do modo de transporte em análise (MA) entre a origem i e o destino $\mathrm{j}$, conforme a matriz O/D;

$V_{i j}^{M A}: \quad$ Vulnerabilidade da rede do modo de transporte em análise (MA) entre a origem i e o destino j.

0 custo de desempenho do modo analisado capta a capacidade dos demais modos de absorver uma demanda extra, caso tenha seus serviços interrompidos, mantendo o nível de serviço em padrões aceitáveis, ao mesmo tempo em que inclui a vulnerabilidade do modo analisado. Logo, quanto maior o custo de desempenho, menor será a resiliência.

Assim, a primeira fração $\left(\frac{D_{i j}^{M A}}{\sum_{m \neq M A} C E X_{i j}^{m}}\right)$ capta a capacidade dos demais modos em absorver a demanda do modo em análise. A segunda porção $\left(^{\frac{\sum_{m \neq M A} t_{i j}^{m}}{m_{i j}-1}} / t_{i j}^{M A}\right)$ agrega o custo do tempo, considerando a variação relativa do tempo de viagem devido à mudança do modo de transporte. Enfim, a terceira parte $\left(V_{i j}^{M A}\right)$ reflete a vulnerabilidade da rede, ou seja, a capacidade da rede em manter a continuidade da operação. A Tabela 3 resume as características e composição das variáveis da Equação 1. Assim, com a finalidade de expressar o desempenho dos sistemas de transportes entre um par de origem (i) e o destino (j), o custo de desempenho de cada modo são somados gerando o custo de desempenho do sistema entre i e j (Equação 2).

$$
C D_{i j}=\sum_{M A} C D_{i j}^{M A}
$$

onde: $\quad C D_{i j}$ : $\quad$ Custo de Desempenho Total do sistema de transportes entre a origem i e o destino j;

$C D_{i j}^{M A}$ : $\quad$ Custo de Desempenho do modo de transporte MA analisado entre a origem i e o destino j.

$$
R_{i j}=e^{-C D_{i j}}
$$

Por fim, com o propósito de normalizar o valor da resiliência entre 0 e 1, adotou-se a função exponencial natural. Dessa forma, a resiliência entre duas zonas (Resiliência Relativa) é calculada por meio da potenciação do simétrico do custo de desempenho na base natural, seguindo a Equação 3. Na Equação 3, apresenta-se a relação inversamente proporcional entre o custo de desempenho e a resiliência. Logo, quanto maior o custo de desempenho, menor será a resiliência e vice-versa, sendo que esta última varia entre 0 e 1. 
Tabela 3 - Descrição dos elementos que compõem a equação1

\begin{tabular}{|c|c|}
\hline Elementos & Definições \\
\hline $\mathrm{N}$ & Número de regiões/zonas em que a cidade foi subdividida \\
\hline $\mathrm{m}$ & $\begin{array}{l}\text { Modos de transporte, onde } m \text { = rodoviário individual, rodoviário coletivo, metroferroviário e } \\
\text { outros }\end{array}$ \\
\hline$m_{i j}$ & Número de modos de transporte entre 2 regiões \\
\hline$c_{i j}^{m}$ & É um caminho entre a origem i e o destino j por meio do modo de transporte $\mathrm{m}$ \\
\hline \multicolumn{2}{|r|}{ Valores de entrada e intermediários } \\
\hline$D_{m}^{i j}$ & $\begin{array}{l}\text { Demanda de viagens do modo de transporte } m \text { entre a origem i e o destino } \mathrm{j} \text { de acordo com a } \\
\text { matriz O/D }\end{array}$ \\
\hline$t_{i j}^{m}(c)$ & Tempo de viagem do caminho $\mathrm{c}$ do modo de transporte $\mathrm{m}$ entre a origem $\mathrm{i}$ e o destino $\mathrm{j}$ \\
\hline$t_{i j}^{m}=\frac{\sum_{c=1}^{p_{i j}^{m}} t_{i j}^{m}(c)}{p_{i j}^{m}}$ & Tempo médio de viagem do modo de transporte $m$ entre a origem i e o destino j \\
\hline$p_{i j}^{m}$ & $\begin{array}{l}\text { Quantidade de caminhos disjuntos (rotas alternativas sem interseção) entre a origem i e o } \\
\text { destino j por meio do modo de transporte m; equivalente ao somatório de caminhos } c_{i j}^{m}\end{array}$ \\
\hline$V_{i j}^{m}=1 / p_{i j}^{m}$ & Vulnerabilidade do modo de transporte MODO entre a origem i e o destino j \\
\hline$V O L_{i j}^{m}(c)$ & $\begin{array}{l}\text { Volume médio de passageiros em hora de pico do caminho c do modo de transporte m entre } \\
\text { a origem i e o destino j }\end{array}$ \\
\hline$V O L_{i j}^{m}=\sum_{c=1}^{p_{i j}^{m}} V O L_{i j}^{m}(c)$ & $\begin{array}{l}\text { Volume médio total de passageiros em hora pico do modo de transporte } m \text { entre a origem i e } \\
\text { o destino j }\end{array}$ \\
\hline$C A P_{i j}^{m}(c)$ & Capacidade do caminho $\mathrm{c}$ modo de transporte $\mathrm{m}$ entre a origem i e o destino $\mathrm{j}$ \\
\hline$C A P_{i j}^{m}=\sum_{c=1}^{p_{i j}^{m}} C A P_{i j}^{m}(c)$ & Capacidade total do modo de transporte $\mathrm{m}$ entre a origem i e o destino $\mathrm{j}$ \\
\hline$C E X_{i j}^{m}$ & $\begin{array}{l}\text { Capacidade média residual do modo de transporte } m \text { entre a origem i e o destino j (diferença } \\
\text { entre a capacidade total e a demanda existente no sistema) }\end{array}$ \\
\hline
\end{tabular}

\subsection{Etapa 4: Cálculo da Resiliência de uma região}

Para avaliação da resiliência de uma região, tomou-se como base a estrutura lógica aplicada para analisar a acessibilidade entre regiões, visto que, em ambos os casos, os indicadores que compõe o índice são quantificados entre pares de origem-destino, estruturando a "Resiliência Relativa" entre as regiões. A partir da agregação das resiliências entre uma determinada região e as demais da cidade, a "Resiliência Integral" dessa região é mensurada. Por fim, a "Resiliência Global" da cidade é caracterizada pelo agrupamento das resiliências de cada região da cidade. Assim, tendo como base as formulações apresentadas por Allen et al. (1993) usadas para avaliar a acessibilidade numa região, considera-se que a resiliência em sistemas de transportes de uma cidade pode ser analisada por meio das formulações definidas pelas Equações 4 e 5.

$$
\begin{aligned}
& R_{i}=\frac{\sum_{j \neq i}^{N} R_{i j}}{N-1} \\
& R=\frac{\sum_{i}^{N} \sum_{j \neq i}^{N} R_{i j}}{N(N-1)}
\end{aligned}
$$

onde: $\quad R_{i j}$ : $\quad$ Resiliência relativa entre as regiões i e j;

$R_{i}$ : $\quad$ Resiliência integral da região i;

$R: \quad$ Resiliência Global da área/cidade em análise;

$R: \quad \quad \quad$ úmero de regiões/zonas em que a cidade foi subdividida.

Portanto, a partir da resiliência relativa entre regiões, a resiliência integral de uma região e a resiliência global de toda a área estudada pode ser avaliada por meio das equações 4 e 5 , respectivamente. Desse modo, a resiliência pode ser comparada para a identificação das áreas que requerem uma maior atenção. 


\subsection{Etapa 5: Determinação do nível de resiliência}

Visando classificar a resiliência qualitativamente, na Tabela 4 é apresentada uma proposta de categorização em seis níveis, analogamente ao HCM (Highway Capacity Manual). Logo, com base nos valores do Índice de Resiliência (Relativa, Integral ou Global), determina-se o nível de resiliência da região analisada.

Tabela 4 - Proposta de Níveis de Resiliência

\begin{tabular}{llll}
\hline $\begin{array}{l}\text { Nível de } \\
\text { Resiliência }\end{array}$ & $\begin{array}{l}\text { Índice de } \\
\text { Resiliência }\end{array}$ & Classificação & Características \\
\hline A & $R \geq 0,9$ & Ótima & $\begin{array}{l}\text { Neste nível, uma pequena perturbação na rede seria facilmente } \\
\text { absorvida } \\
\text { Uma leve perturbação na rede seria absorvida com uma pequena } \\
\text { alteração na mobilidade } \\
\text { Uma pequena falha no sistema afetaria a rede localmente, pouco } \\
\text { afetando a mobilidade }\end{array}$ \\
$\mathrm{C}$ & $0,8 \leq \mathrm{R}<0,9$ & Muito boa & $\begin{array}{l}\text { Existe uma aproximação da instabilidade e quando pequenas } \\
\text { perturbações já afetam a mobilidade } \\
\text { Qualquer alteração no sistema, afetará a mobilidade significativamente } \\
\text { Uma pequena perturbação no sistema pode resultar em um grande } \\
\text { colapso na rede. }\end{array}$ \\
\hline
\end{tabular}

\section{APLICAÇÃO}

Para um melhor entendimento do procedimento, apresenta-se uma aplicação em que se procurou avaliar a resiliência relativa entre regiões da cidade do Rio de Janeiro e o centro da cidade.

\subsection{Etapa 1: Reconhecimento da Rede}

Para o estudo de caso, a definição das sub-regiões do Rio de Janeiro segue uma adaptação das áreas de planejamento da Região de acordo com o PDTU (Plano Diretor de Transporte Urbano) da Região Metropolitana do Rio de Janeiro (PDTU, 2016). Ao todo, são cinco áreas de planejamento (AP): a AP1, região central, a AP2, que compreende a Zona Sul e a Grande Tijuca, a AP3, que consiste no restante da zona norte, a AP4, que é definida pelas regiões administrativas da Barra da Tijuca e de Jacarepaguá, e a AP5, integrando o restante da zona oeste. Entretanto, como a Floresta da Tijuca representa uma segregação significativa entre a Grande Tijuca e a zona sul, essas áreas foram divididas em AP2-Tijuca e AP2-Zona Sul para a aplicação do procedimento, conforme apresentado na Figura 1.

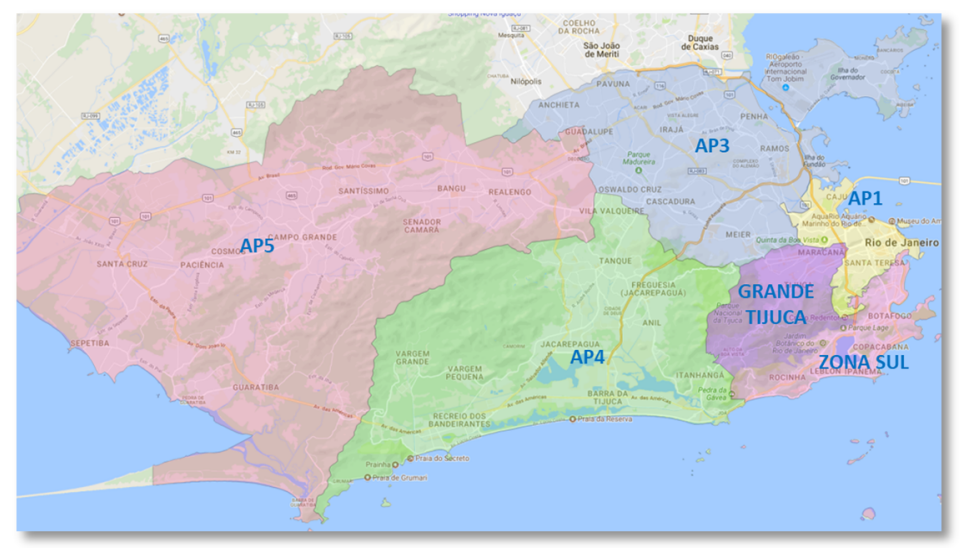

Figura 1. Representação das regiões administrativas 
Para o cálculo dos indicadores e do índice de resiliência de transportes do Rio de Janeiro, consideraram-se os três modos existentes (rodoviário individual, rodoviário coletivo e metroferroviário), sendo todos os indicadores mensurados para cada par origem-destino, a partir de dados do PDTU 2016.

\subsection{Etapa 2: Medição dos Indicadores}

A partir da definição dos centróides de cada uma das seis zonas de análise, identificaram-se os possíveis caminhos/rotas disjuntos entre cada região e a região central (AP1) por modo. Esta etapa é fundamental para o cálculo da vulnerabilidade. Na Figura 2, são apresentados, como exemplo, os caminhos disjuntos (rotas alternativas) pelo modo rodoviário individual entre o centróide da AP2-Zona Sul e o centróide da AP1 (Centro). As variáveis Tempo de Viagem, Capacidade e Demanda para cada um desses caminhos são apresentadas na Tabela 5.

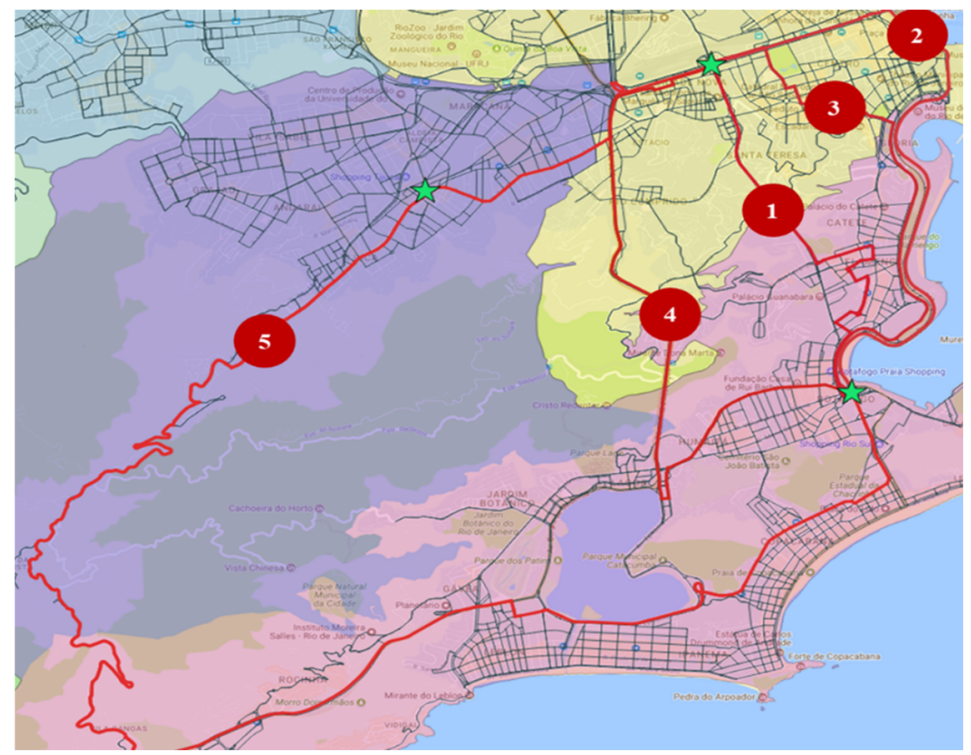

Figura 2. Caminhos disjuntos entre Zona Sul e AP1

Tabela 5 - Variáveis dos caminhos disjuntos do modo rodoviário individual entre a Zona Sul e a AP1 na hora de pico

\begin{tabular}{lllllll}
\hline ORIGEM & DESTINO & Caminho & Tempo & Capacidade Média (veículos) & Distância & Volume de veículos \\
\hline AP2-Zona Sul & AP1 & 1 & 10,12 & 2067,0 & 7,37 & 1125,8 \\
& 2 & 10,84 & 4799,2 & 9,96 & 2260,2 \\
& 3 & 12,28 & 3027,0 & 8,08 & 1370,8 \\
& & 20,82 & 2732,4 & 10,79 & 1795,5 \\
& & 5 & 59,90 & 2744,1 & $33, .38$ & 1315,5 \\
\hline
\end{tabular}

Como pode ser observado na Tabela 5, o caminho de número 5 apresenta distância e tempo de viagem significativamente maiores que os demais. Portanto, em situações semelhantes a essa, optou-se por excluir caminhos com valores cujos tempos de deslocamento e distância sejam três vezes ou mais superiores ao a pior rota considerada. Dessa forma, considerando a possibilidade de 4 caminhos/rotas alternativos, a vulnerabilidade do modo rodoviário entre a zona AP2-zona sul e a AP1- centro é $0,25(V=1 / 4)$.

Assim, os indicadores de Vulnerabilidade da Rede, Tempo Médio de Viagem, Capacidade e Volume de Passageiros foram quantificados da seguinte forma: 
a) Vulnerabilidade, considerando 4 caminhos alternativos:

$$
V_{i j}^{m}=\frac{1}{p_{i j}^{m}}(6) \quad V_{\text {ZonaSul }-A P 1}^{\text {ROD IND }}=\frac{1}{4}=0,25
$$

b) Tempo médio de viagem do transporte individual na hora de pico:

$$
t_{i j}^{m}=\frac{\sum_{c=1}^{p_{i j}^{m}} t_{i j}^{m}(c)}{p_{i j}^{m}}(7) \quad t_{\text {ZonaSul }-A P 1}^{\text {ROD IND }}=\frac{10.12+10.84+12.28+20.82}{4}=13.51 \mathrm{~min}
$$

c) Capacidade Total do modo individual na hora de pico

$$
\begin{gathered}
C A P_{i j}^{m}=\sum_{c=1}^{p_{i j}^{m}} C A P_{i j}^{m}(c)(8) \\
C A P_{\text {Zonasul-AP1 }}^{\text {RODIND }}=(2067+4799+3027+2732)=12625 \text { veículos } \sim 16792 \text { passageiros }\left({ }^{*}\right)
\end{gathered}
$$

d) Volume de passageiros no transporte individual na hora de pico

$$
\begin{gathered}
V O L_{i j}^{m}=\sum_{c=1}^{p_{i j}^{m}} V_{O} O L_{i j}^{m}(c)(9) \\
V O L_{\text {Zonasul-AP1 }}^{\text {RODIND }}=1126+2260+1371+1796=6552 \text { veículos } \sim 8715 \text { passageiros }
\end{gathered}
$$

$\left(^{*}\right)$. Considerou-se 1,3 passageiros por veículo, conforme sugestão do PDTU.

\subsection{Etapas 3, 4 e 5: Método de Cálculo do Índice, Cálculo da Resiliência de uma região e Determinação do nível de resiliência}

Ao final da quantificação dos indicadores, prosseguiu-se ao cálculo do Custo de Desempenho $\left(C D^{M A}{ }^{i j}\right)$ para cada modo de transporte, empregando a Equação 1, e, em seguida, a quantificação do Custo Total de Desempenho $\left(C D^{i j}\right)$, a Resiliência Relativa $\left(R^{i j}\right)$ entre as zonas e Resiliência

\begin{tabular}{|c|c|c|c|c|c|c|}
\hline \multirow{2}{*}{ MODOS } & ORIGEM & TIJUCA & ZONA SUL & AP3-ZONA NORTE & AP4-BARRA & AP5-ZONA OESTE \\
\hline & DESTINO & \multicolumn{5}{|c|}{ CENTRO } \\
\hline \multirow{8}{*}{ 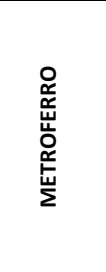 } & DEMANDA & 1023 & 2385 & 7272 & 1191 & 1533 \\
\hline & VOLUME MÉDIO & 7652 & 16302 & 20616 & 13245 & 11651 \\
\hline & CAPACIDADE & 15913 & 35909 & 31743 & 15913 & 15597 \\
\hline & CAPACIDADE EXTRA (OUTROS MODOS) & 24225 & 40935 & 55148 & 16573 & 14052 \\
\hline & TEMPO & 8.86 & 14.90 & 50.17 & 47.44 & 83.06 \\
\hline & TEMPO MÉDIO - OUTROS MODOS & 25.56 & 25.75 & 59.29 & 73.83 & 122.71 \\
\hline & VULNERABILIDADE & 1.00 & 1.00 & 0.50 & 1.00 & 1.00 \\
\hline & CUSTO DE DESEMPENHO & 0.1218 & 0.1007 & 0.0779 & 0.1118 & 0.1611 \\
\hline \multirow{8}{*}{ 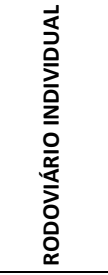 } & DEMANDA & 4172 & 11084 & 6099 & 1832 & 638 \\
\hline & VOLUME & 9714 & 8715 & 9638 & 7162 & 6201 \\
\hline & CAPACIDADE & 15685 & 16792 & 16605 & 12164 & 11019 \\
\hline & CAPACIDADE EXTRA (OUTROS MODOS) & 26516 & 27684 & 59308 & 14239 & 13181 \\
\hline & TEMPO & 18.49 & 13.51 & 45.83 & 55.66 & 93.36 \\
\hline & TEMPO MÉDIO - OUTROS MODOS & 20.75 & 26.44 & 61.46 & 69.72 & 117.56 \\
\hline & VULNERABILIDADE & 0.25 & 0.25 & 0.25 & 0.33 & 0.33 \\
\hline & CUSTO DE DESEMPENHO & 0.0441 & 0.1033 & 0.0345 & 0.0537 & 0.0203 \\
\hline \multirow{8}{*}{ 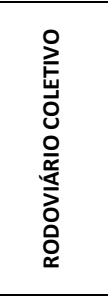 } & DEMANDA & 7908 & 16650 & 49770 & 8151 & 10488 \\
\hline & VOLUME & 15805 & 16924 & 35276 & 7602 & 4487 \\
\hline & CAPACIDADE & 34060 & 49781 & 83457 & 19174 & 13722 \\
\hline & CAPACIDADE EXTRA (OUTROS MODOS) & 14231 & 27684 & 18094 & 7671 & 8764 \\
\hline & TEMPO & 32.64 & 37.99 & 72.75 & 92.00 & 152.06 \\
\hline & TEMPO MÉDIO - OUTROS MODOS & 13.67 & 14.21 & 48.00 & 51.55 & 88.21 \\
\hline & VULNERABILIDADE & 0.25 & 0.25 & 0.25 & 0.33 & 0.33 \\
\hline & CUSTO DE DESEMPENHO & 0.0582 & 0.0562 & 0.4537 & 0.1985 & 0.2314 \\
\hline
\end{tabular}
Integral $\left(R^{i}\right)$ da Região AP1 (centro), utilizando as Equações 2, 3 e 4, respectivamente. Os valores referentes aos indicadores e ao custo de desempenho por modo de transporte entre cada uma das zonas de tráfego, já considerando as rotas principal e alternativas entre cada zona e a zona AP1-centro, estão expostos na Tabela 6.

Tabela 6 - Indicadores para Quantificação da Resiliência entre AP1 e demais zonas 
Tabela 6 - Indicadores para Quantificação da Resiliência entre AP1 e demais zonas (continuação)

\begin{tabular}{|c|c|c|c|c|c|c|}
\hline \multirow{2}{*}{ MODOS } & ORIGEM & TIJUCA & ZONA SUL & AP3-ZONA NORTE & AP4-BARRA & AP5-ZONA OESTE \\
\hline & DESTINO & \multicolumn{5}{|c|}{ CENTRO } \\
\hline & DEMANDA & 1023 & 2385 & 7272 & 1191 & 1533 \\
\hline & CUSTO TOTAL & 0.2242 & 0.2603 & 0.5661 & 0.3640 & 0.4129 \\
\hline & RESILIÊNCIA RELATIVA & 0.7992 & 0.7708 & 0.5677 & 0.6949 & 0.6618 \\
\hline & NÍVEL DE RESILIÊNCIA & $\mathrm{C}$ & $\mathrm{C}$ & $\mathrm{E}$ & $D$ & $D$ \\
\hline
\end{tabular}

O resultado mostrou que a resiliência relativa entre a zona AP3 e a região central (AP1) apresenta o pior resultado em relação às outras zonas, com uma resiliência instável de nível E, ou seja, qualquer ruptura/perturbação no sistema irá afetar significativamente a mobilidade entre essas regiões. A melhor resiliência relativa é apresentada entre a Tijuca e o Centro (AP1), expressando um nível C, equivalente a uma boa resiliência. Enfim, a resiliência integral da zona central (AP1) é calculada empregando a Equação 4. Assim, verifica-se que a Área de Planejamento 1, caracterizada pela região central, apresenta uma Resiliência Integral de 0.6989, de modo que apresenta uma resiliência integral nível D.

\section{ANÁLISE DE SENSIBILIDADE DO ÍNDICE}

Na aplicação do procedimento, foram estudados pares OD que são atendidos por três modos de transporte, conforme a situação existente no estudo de caso. Assim, com a finalidade de avaliar a consistência do índice proposto, considerou-se um cenário onde apenas dois modos são ofertados em um determinado par OD. Desse modo, o procedimento foi aplicado supondo que apenas o modo rodoviário coletivo e o individual estão disponíveis na cidade. Dois cenários são analisados: (i) quando a demanda do modo rodoviário coletivo permanece inalterada; (ii) considerando que a demanda do modo rodoviário coletivo inclui toda a demanda relativa ao modo metroferroviário. Na Tabela 7 são apresentados os resultados dessa análise.

Tabela 7 - Comparação da Resiliência Relativa entre os cenários

\begin{tabular}{llllllll}
\hline \multirow{2}{*}{ ORIGEM } & \multirow{2}{*}{ DESTINO } & \multicolumn{2}{l}{ Resiliência Relativa } & & & & \\
\cline { 3 - 8 } & & Cenário Original & NR & Cenário I & NR & Cenário II & NR \\
\hline AP2-TIJUCA & & 0.7992 & $\mathrm{C}$ & 0.7494 & $\mathrm{C}$ & 0.7315 & $\mathrm{C}$ \\
AP2-ZONA SUL & & 0.7708 & $\mathrm{C}$ & 0.6568 & $\mathrm{D}$ & 0.6398 & $\mathrm{D}$ \\
AP3-ZONA NORTE & \multirow{2}{*}{ CENTRO } & 0.5677 & $\mathrm{E}$ & 0.3087 & $\mathrm{~F}$ & 0.2619 & $\mathrm{~F}$ \\
AP4-BARRA & & 0.6949 & $\mathrm{D}$ & 0.6598 & $\mathrm{D}$ & 0.6288 & $\mathrm{D}$ \\
AP5-ZONA OESTE & & 0.6618 & $\mathrm{D}$ & 0.6169 & $\mathrm{D}$ & 0.578 & $\mathrm{E}$ \\
\hline
\end{tabular}

No cenário exploratório I, foram excluídos todos os indicadores referentes ao modo metroferroviário, não alterando os valores dos outros modos (apenas os indicadores construídos a partir dos indicadores dos outros modos foram modificados, como capacidade extra e tempo médio nos outros modos). Percebe-se uma diminuição da resiliência relativa entre as regiões para esse cenário.

O cenário II foi gerado de forma a tornar a comparação mais fidedigna. Observa-se que esse cenário revela uma redução ainda maior na Resiliência Relativa entre regiões, certificando a coerência do índice em situações onde existem apenas dois modos de transporte entre zonas de uma cidade. 0 objetivo dessa verificação foi atestar a aplicabilidade do índice proposto, mostrando que, apesar do índice ser resultado de um somatório de custos de desempenho dos 
modos, o custo total de desempenho será maior quanto menor a quantidade de modos ofertados e, consequentemente, a resiliência será menor.

\section{CONSIDERAÇÕES FINAIS}

Este trabalho, ao desenvolver um procedimento para quantificar e qualificar a resiliência própria de sistemas de transportes em condições pré-evento, fornece assistência a planejadores e gestores na verificação das regiões que seriam mais afetadas diante de situações de emergências, assim como na identificação de questões que necessitam de mais atenção com o intuito de reduzir o impacto na mobilidade. Desse modo, o estudo oferece uma ferramenta para a definição de estratégias preventivas como alternativa às corretivas, que geralmente requerem mais aporte de capital. Ressalta-se que o valor de resiliência resultante de cada região oferece, principalmente, a possibilidade de comparar as regiões da cidade e verificar aquelas que têm a menor resiliência, indicando a necessidade de priorizar os investimentos nessas localidades.

0 procedimento desenvolvido pode ser aplicado em qualquer cidade e suas sub-regiões, estando sujeito à forma como os dados são viabilizados, podendo ser adaptados conforme necessidade. Neste artigo, o procedimento foi aplicado para a avaliação da resiliência relativa considerando os sistemas de transportes do Município do Rio de Janeiro, que foi divido em seis subregiões para a análise da resiliência entre zonas. Os resultados se mostraram coerentes com o conceito adotado de resiliência própria do sistema, isto é, a capacidade em manter as condições de deslocamento em padrões aceitáveis, já que a resiliência é maior quanto maior é a capacidade dos modos alternativos em absorver a demanda não atendida de um determinado modo quando este tem sua operação impactada ao mesmo tempo em que provêm os serviços em padrões desejáveis.

\section{REFERÊNCIAS}

Adams, T. M.; K. R. Bekkem e E. J. Toledo-Durán (2012) Freight Resilience Measures. Journal of Transportation Engineering, v. 138, n. 11, P. 1403-1409. DOI:10.1061/(ASCE)TE.1943-5436.0000415

Adjetey-Bahun, K.; B. Birregah; E. Châtelet e J. L. Planchet (2016) A model to quantify the resilience of mass railway transportation systems. Reliability Engineering \& System Safety, v. 153, p. 1-14. D0I:10.1016/j.ress.2016.03.015

Allen, B.; D. Liu e S. Singers (1993) Accessibility Measures of U.S. Metropolitan Areas. Transportation Research Part B: Methodological, v. 278, n. 6, p. 439-449. DOI:0191-2615/93

Arcidiacono, V.; G. P. Cimellaro; A. M. Reinhorn e M. Bruneau (2012) Community resilience evaluation including interdependencies. 15th World Conference on Earthquake Engineering. Sociedade Portuguesa de Engenharia Sismica, Lisboa, POR. Disponível em: <http://www.iitk.ac.in/nicee/wcee/article/WCEE2012_5532.pdf>. (Acesso em 01/01/2017).

Ázaro, É. S. (2017) Resiliência dos Serviços de Ônibus Urbanos a Inundações: Uma Proposta de Indicador para a Cidade do Rio de Janeiro. Dissertação (Mestrado em Ciências) - Universidade Federal do Rio de Janeiro, Programa de Pós-Graduação e Pesquisa de Engenharia (COPPE), Engenharia de Transportes. Disponível em : <http://www.pet.coppe.ufrj.br/index.php/pt/producao-academica/dissertacoes/2017/341-resiliencia-dos-servicos-de-onibus-urbanos-a-inundacoesuma-proposta-de-indicador-para-a-cidade-do-rio-de-janeiro>. (Acesso em 06/08/2018).

Azolin, L. G. e A. N. R. Silva (2019) O Transporte Público em uma Estratégia de Avaliação da Resiliência na Mobilidade Urbana. $33^{\circ}$ Congresso de Pesquisa e Ensino em Transporte da ANPET.

Balal, E.; G. Valdez; J. Miramontes; M. Mercado e R. L. Cheu (2017) Urban Transportation Network Resilience: Comparative Evaluation of Measures. TRB 2017 Annual Meeting. Transportation Research Board, Washington, D.C.

Beiler, M. O.; S. Mcneil; D. Ames e R. Gayley (2013) Identifying Resiliency Performance Measures for Megaregional Planning Case Study of the Transportation Corridor Between Boston, Massachusetts, and Washington, DC. Transportation Research Record, v. 2397, p. 153-160. DOI:10.3141/2397-18

Bruneau, M.; S. E. Chang; R. T. Eguchi; G. C. Lee; T. D. O'rourke; A. M. Reinhorn; M. Shinozuka; K. Tierney; W. A. Wallace e D. Von Winterfeldt (2003) A Framework to Quantitatively Assess and Enhance the Seismic Resilience of Communities. Earthquake Spectra, v. 19, n. 4, p. 733-752. DOI:10.1193/1.1623497

Cartalis, C. (2014) Toward resilient cities - a review of definitions, challenges and prospects. Advances In Building Energy Research, v. 8, n. 2, p. 259-266. DOI:10.1080/17512549.2014.890533

Chan, R. e J. L. Schofer (2016) Measuring Transportation System Resilience : Response of Rail Transit to Weather Disruptions. Natural Hazards Review, v. 17, n. 1, p. 1-8. DOI:10.1061/(ASCE)NH 
Chen, L. e E. Miller-Hooks (2012) Resilience: An Indicator of Recovery Capability in Intermodal Freight Transport. Transportation Science, v. 46, n. 1, p. 109-123. DOI:10.1287/trsc.1110.0376

Comfort, L. (1999) Shared Risk: Complex Systems in Seismic Response. Pergamon, New York.

Cox, A.; F. Prager e A. Rose (2011) Transportation security and the role of resilience: A foundation for operational metrics. Transport Policy, v. 18, n. 2, p. 307-317. DOI:10.1016/j.tranpol.2010.09.004

D'Lima, M. e F. Medda (2015) A new measure of resilience: An application to the London Underground. Transportation Research Part A: Policy and Practice, v. 81, p. 35-46. DOI:10.1016/j.tra.2015.05.017

Donovan, B. e D. B. Work (2017) Empirically quantifying city-scale transportation system resilience to extreme events. Transportation Research Part C: Emerging Technologies, v. 79, p. 333-346. DOI:10.1016/j.trc.2017.03.002

Faturechi, R. e E. Miller-Hooks (2014) Measuring the performance of transportation infrastructure systems in disasters: A comprehensive review. Asce Journal of Infrastructure Systems, v. 21, n. 1, p. 1-15. D0I:10.1061/(ASCE)IS.1943555X.0000212.

Fernandes, V. A.; R. Rothfuss; V. Hochschild; W. R. Da Silva e M. P. S. Santos (2017) Resiliência da mobilidade urbana: uma proposta conceitual e de sistematização. Transportes, v. 25, n. 4, p. 147. DOI:10.14295/transportes.v25i4.1079

Freckleton, D.; K. Heaslip; W. Louisell e J. Collura (2012) Evaluation of Resiliency of Transportation Networks After Disasters. Transportation Research Record, v. 5, p. 109-116. DOI:10.3141/2284-13

Godschalk, D. R. (2003) Urban Hazard Mitigation: Creating Resilient Cities. Natural Hazards Review, v.4 , n. 3, p. $136-143$. DOI:10.1061/(ASCE)1527-6988(2003)4:3(136)

King, D. e A. Shalaby (2016) Performance Metrics and Analysis of Transit Network Resilience in Toronto. TRB 2016 Annual Meeting. Transportation Research Board, Washington, D. C.

Leu, G.; H. Abbass e N. Curtis (2010) Resilience of ground transportation networks: A case study on Melbourne. 33rd Australasian Transport Research Forum. World Transit Research, Canberra.

Martins, M e A. Silva (2018) Uma estratégia para avaliação da resiliência na mobilidade urbana. Transportes, v. 26, n. 3, p. 7586. DOI:10.14295/transportes.v26i3.1625

Meerow, S.; J. P. Newell e M. Stults (2016) Defining urban resilience: A review. Landscape and Urban Planning, v. 147, p. 38-49. DOI:10.1016/j.landurbplan.2015.11.011

Mileti, D. (1999) Disasters by Design: A Reassessment of Natural Hazards in the United States. Joseph Henry Press, Washington, D. C.

Murray-Tuite, P. M. (2006) A comparison of transportation network resilience under simulated system optimum and user equilibrium conditions. Proceedings - Winter Simulation Conference (p. 1398-1405). Institute of Electrical and Electronics Engineers, Monterey. DOI:10.1109/WSC.2006.323240

Omer, M.; A. Mostashari e R. Nilchiani (2013) Assessing resilience in a regional road-based transportation network. International Journal of Industrial and Systems Engineering, v. 13, n. 4, p. 389-408. D0I:10.1504/IJISE.2013.052605

Ortiz, D.; L. Ecola e H. Willis (2009) Adding Resilience to the Freight System in Statewide and Metropolitan Transportation Plans: Developing a Conceptual Approach.

Pant, S. B. (2012) Transportation Network Resiliency : A Study of self-Annealing. Utah State University. Disponível em: < https://digitalcommons.usu.edu/cgi/viewcontent.cgi?referer=\&httpsredir=1\&article=2435\&context=etd $>$. (Acesso em 01/01/2017).

PDTU. (2016) Relatório 13 - Síntese do PDTU. Governo do Estado do Rio de Janeiro, Rio de Janeiro - RJ.

Rose, A. (2004) Defining and Measuring Economic Resilience to Earthquakes. Disaster Prevention and Management, v. 13, n. 4, p. 307-314. DOI:10.1108/09653560410556528

Rose, A. (2007) Economic resilience to natural and man-made disasters: Multidisciplinary origins and contextual dimensions. Environmental Hazards, v. 7, n. 4, p. 383-398. DOI:10.1016/j.envhaz.2007.10.001

Santos, A. S. (2014) A Importância do Setor de Transportes para o Aumento de Resiliência das Cidades frente à Mudança Climática: Uma Proposta de Plano de Ação para a Cidade do Rio de Janeiro. Tese (Doutorado em Ciências) - Universidade Federal do Rio de Janeiro, Programa de Pós-Graduação e Pesquisa de Engenharia (COPPE), Engenharia de Transportes. Disponível em: < http://www.pet.coppe.ufrj.br/images/documentos/teses/TESE_ANDREA_SOUZA_SANTOS.pdf>. (Acesso em 01/01/2017).

Santos, T. F.; M. A. V. Silva e V. A. Fernandes (2018) Análise da Resiliência Frente a Ausência de Integração Tarifária: 0 Caso do Município do Rio de Janeiro - Brasil. $32^{\circ}$ Congresso de Pesquisa e Ensino em Transporte da ANPET. DOI:10.1017/CBO9781107415324.004

Ta, C.; A. V. Goodchild e K. Pitera (2009) Structuring a Definition of Resilience for the Freight Transportation System. Transportation Research Record, v. 2097, n. 1, p. 19-25. D0I:10.3141/2097-03

Thomé, A. M. T.; L. F. E. Scavarda e A. J. Scavarda (2016) Conducting systematic literature review in operations management. Production Planning \& Control, v. 27, n. 5, p. 408-420. DOI:10.1080/09537287.2015.1129464

Tierney, K. e M. Bruneau (2007) A Key to Disaster Loss Reduction. TR News, 14-18. Disponível em: <http://onlinepubs.trb.org/onlinepubs/trnews/trnews250_p14-17.pdf>. (Acesso em 01/01/2017).

Timmerman, P. (1981) Vulnerability, Resilience and the Collapse of Society: A Review of Models and Possible Climatic Applications. Environmental Monograph. DOI:10.1002/joc.3370010412

VTPI. (2017) Online TDM Encyclopedia - Evaluating Transportation Resilience. Disponível em: <http://www.vtpi.org/tdm/tdm88.htm>. (Acesso em 01/01/2017). 
Vugrin, E. D.; N. J. K. Brown e M. A. Turnquist (2010) Optimal recovery sequencing for critical infrastructure resilience assessment. Sandia National Laboratories, Albuquerque. DOI:10.2172/1007322 